\title{
GRAVOTHERMAL OSCILLATIONS
}

\author{
JUNICHIRO MAKINO \\ Department of Graphics and Information Science, \\ College of Arts and Sciences, University of Tokyo \\ 3-8-1 Komaba, Meguro-ku, Tokyo 153, Japan
}

\begin{abstract}
We present the first clear evidence that the gravothermal oscillation takes place in $N$-body systems. We performed direct $N$-body simulations of systems of point-mass particles with particle numbers from 2,048 to 32,768 . In the simulation with 32,768 particles, the central density shows an oscillation with an amplitude of $\sim 10^{3}$, which is similar to what was observed in more approximate models such as a conducting gas sphere and one-dimensional Fokker-Planck calculations. The amplitude is smaller for a smaller number of particles. The number of particles in the core at the maximum contraction is $\sim 10$ for all runs, while the number of particles at the maximum expansion is about $0.01 N$. For 16,384 - and 32,768 -body runs, the temperature inversion during the expansion phase is clearly visible.
\end{abstract}

\section{Introduction}

Gravothermal oscillation was first found by Sugimoto and Bettwieser (1983), who modelled the post-collapse evolution of globular clusters using a conducting gas sphere with artificial energy production. In their model the energy production is expressed as $\epsilon=C \rho^{k}$, where $\epsilon$ is the energy production per unit mass per unit time and $\rho$ is the density. The power-law index they used is 1 or 2 . They found similar oscillations in both cases, for a wide range of the value of coefficient $C$.

Before they found the oscillation, the standard picture of the evolution of a globular cluster had been the following two-stage one. The first stage is the gravothermal collapse, or core collapse, driven by the gravothermal instability. This collapse leads to a self-similar evolution, in which the core density reaches infinity and the core mass goes down to zero in a finite time 
(Hachisu, et al. 1978, Lynden-Bell and Eggleton 1980).

In a real $N$-body system, contraction is halted by the energy production by 3-body binaries. It had been believed that the whole system expands homologously, driven by the energy generation from binaries after the contraction is halted (Goodman, 1984).

What Sugimoto and Bettwieser (1983) found is that this homologous expansion is unstable in a way similar to the way in which an isothermal sphere is unstable. A linearized stability analysis by Goodman (1987) showed that the expansion is unstable against thermal perturbation if the coefficient for the energy production is small. If translated back to the total number of particles, the system is unstable if $N>7,000$. This result is confirmed also with Fokker-Planck calculations (Cohn et al., 1989).

To determine if such an oscillation actually takes place in real globular clusters, we have to perform a direct $N$-body simulation with a sufficiently large number of particles, since gas models and FP models have many simplifying assumptions that might make the evolution completely different. For example, both assume spherical symmetry, while in $N$-body simulation the core is known to wander around (Makino and Sugimoto 1987, Heggie et al. 1994). Both assume that the energy production by binaries is smooth, while in an $N$-body system binaries are formed stochastically.

An $N$-body simulation with sufficiently large number of particles has been impossible, simply because the requirement for computer power has been excessive. The largest simulation which has been tried on a generalpurpose supercomputer is 10,000-body run by Spurzem and Aarseth (1996), which does not cover a long enough time after the first collapse.

In the present paper, we describe the result of $N$-body simulations with number of particles 2,048-32,768. All simulations were performed on GRAPE-4, a special-purpose computer for collisional $N$-body simulation. Our main result is the following. First, for large $N$, the core density and core mass exhibited an oscillation of large amplitude. The core mass at maximum expansion is almost independent of the number of particles, and is in good agreement with FP or gas model results (1-2\% of the total mass). Second, we confirmed that the observed oscillation is driven by the gravothermal instability. There were several long expanding periods without any energy input. The temperature inversion is visible in such expansion phases. In addition, the behavior of the core density is strikingly similar to the result of FP calculations with a stochastic heat source (Takahashi and Inagaki 1991), suggesting that the mechanism is the same.

The structure of this paper is the following. In section 2, we describe the initial model, the numerical method and the computer used. In section 3 we present the results. Section 4 is for discussion. 


\section{Model and Numerical method}

\subsection{INITIAL MODELS AND THE SYSTEM OF UNITS}

We followed the evolution of isolated systems of point-mass particles. For all calculations, we used random realization of the Plummer model as the initial condition. We used the standard system of units (Heggie and Mathieu 1986), in which $G=1, M=1$, and $E=-1 / 4$, where $G$ is the gravitational constant, $M$ and $E$ are the total mass and the total energy of the cluster. All particles have the same mass $m=1 / N$, where $N$ is the total number of particles. The half-mass crossing time $t_{h c}$ is $2 \sqrt{2}$ in this unit.

\subsection{NUMERICAL METHOD}

For all calculations, we used NBODY4 (Aarseth 1995), modified for GRAPE4 (Taiji et al. 1995). The numerical integration scheme adopted in NBODY4 is the 4th order Hermite scheme (Makino and Aarseth 1992). It implements the hierarchical (block) timestep algorithm (McMillan 1986, Makino 1991) to use the GRAPE hardware efficiently. Close two-body encounters and stable binaries are handled by KS regularization (Kustaanheimo and Stiefel 1965). Special treatment for compact few-body subsystems is also possible.

The outputs are taken at intervals of a fixed time, which is some fraction of the crossing time. We recorded the central density, core radius, number of particles in the core, the radii of Lagrangian shells, the velocity dispersion within Lagrangian shells, the binaries and their binding energies. The core parameters are calculated following Casertano and Hut (1985).

The accuracy of the time integration is adjusted so that the energy error between two outputs is smaller than a certain prescribed value. The value we used is $1 \times 10^{-5} \sim 1 \times 10^{-6}$ depending on $N$. We required higher accuracy for larger $N$, since the duration of the simulation is longer. When the energy error is very large, the program automatically reads the output at the previous checkpoint and restarts with a reduced accuracy parameter.

\subsection{HARDWARE}

For all calculations, we used GRAPE-4 (Taiji 1995). The GRAPE-4 is a special-purpose computer designed to accelerate the $N$-body simulation using the Hermite integrator and hierarchical timestep algorithm. The fully configured system has a theoretical peak speed of 1.08 Tflops. The simulations reported in the present paper were performed while the assembly and testing of the GRAPE-4 system were under way. Thus the number of processors varies during the calculation. For most of the $32 \mathrm{k}$ particle run, we used one quarter of the machine which has a peak speed of 270 Gflops. 


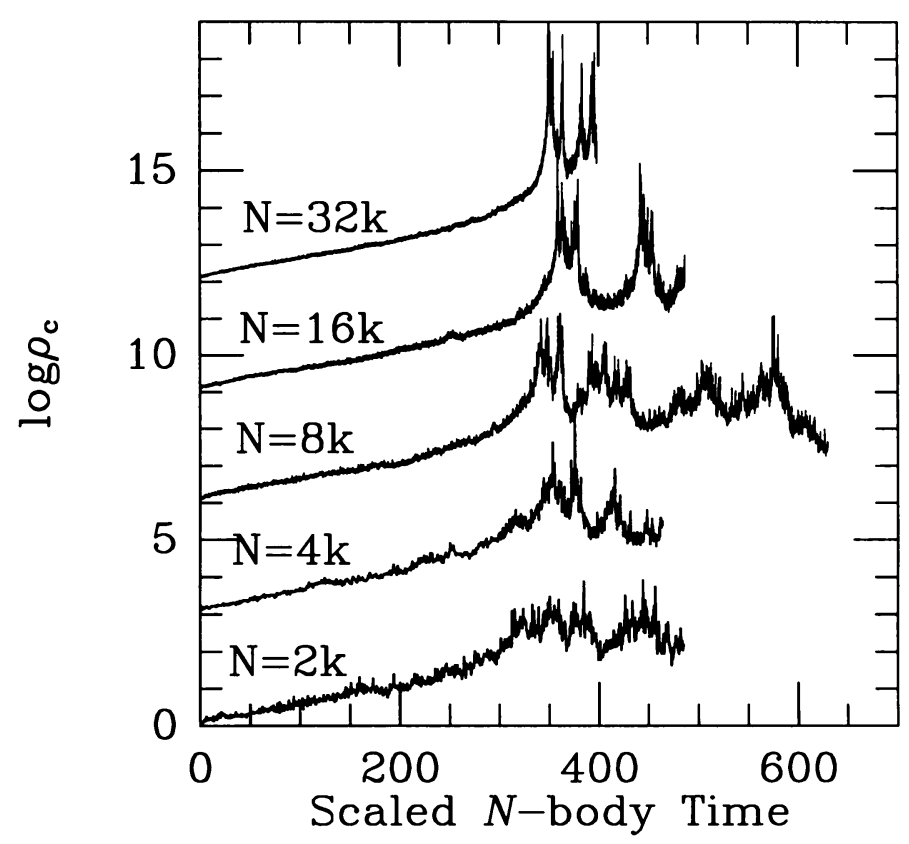

Figure 1. The logarithm of the central density plotted as a function of the scaled $N$-body time. Curves for different values of $N$ are vertically shifted by 3 units.

\section{Result}

\subsection{CORE PARAMETERS}

Figure 1 shows the time evolution of the central density for all runs. The time is scaled so that the thermal timescale is same for all runs. The scaling factor is $t_{r}(1000) / t_{r}(N)=212.75 \log (0.11 N) / N$ (Giersz and Heggie 1994). The core density shows an oscillation with large amplitude in calculations with large $N(>16 \mathrm{k})$. No matter what is the real nature of this oscillation, it is at least clear that the core density of the $N$-body system shows oscillation with the amplitude comparable to that observed in gas models or FP calculations. For runs with small $N(2 \mathrm{k}$ and $4 \mathrm{k})$, there are some oscillationlike features but they are hardly distinguishable from fluctuations. For large values of $N$, however, the oscillation with large amplitude is clearly visible.

Note that in figure 1 there is no clear transition from stable expansion to oscillation or from regular oscillation to chaotic oscillation, which were observed in gas and FP models (e.g. Cohn et al. 1989, Heggie and Ramamani 1989). The reason is that binaries emit energy intermittently at the formation time and as a result of binary-single-body interactions.

Takahashi and Inagaki (1991) incorporated this stochastic nature of the 

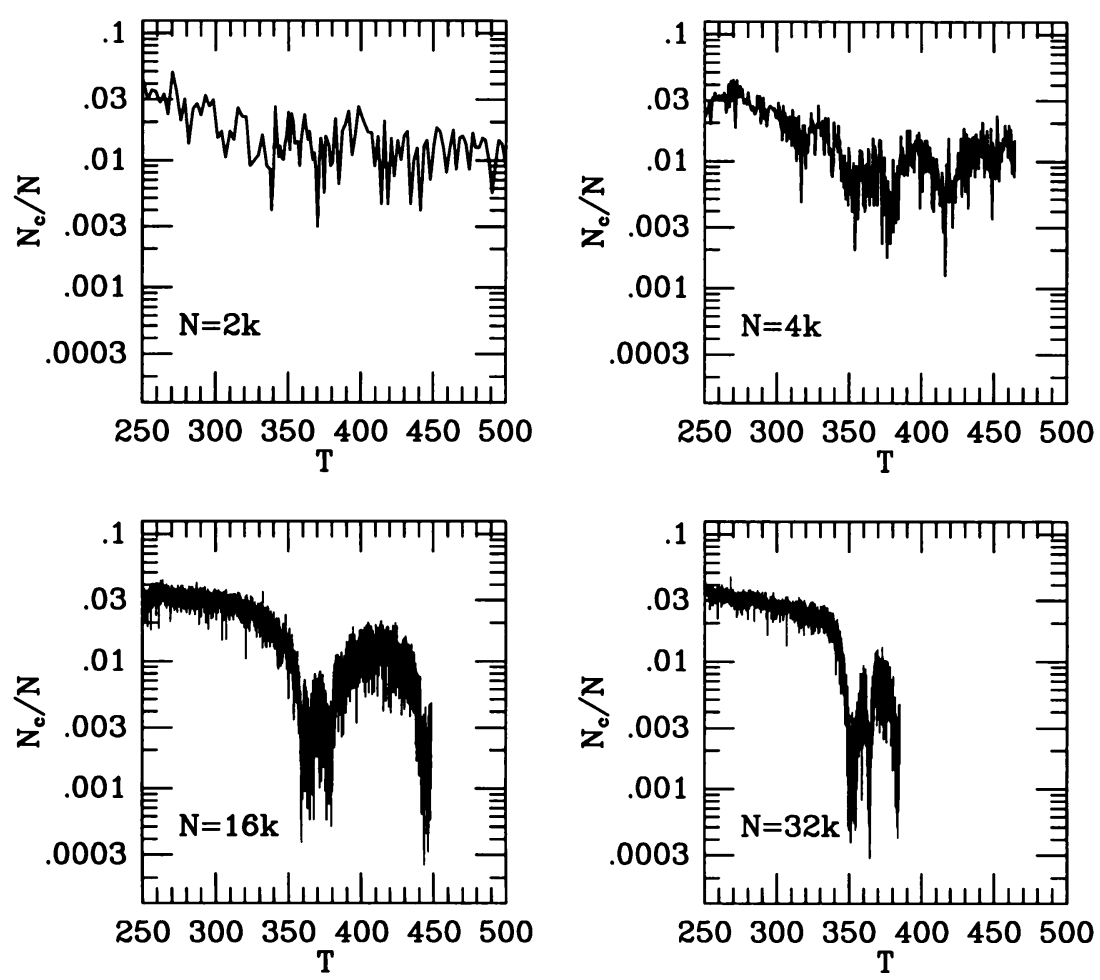

Figure 2. The number of particles in the core as the function of the scaled time, for simulations with $2 \mathrm{k}, 4 \mathrm{k}, 16 \mathrm{k}$, and $32 \mathrm{k}$ particles

energy source into their FP model and found that the core density shows chaotic oscillatory behavior even if the energy production rate is larger than the critical value. They also found that the amplitude of the oscillation is smaller for larger energy input (smaller $N$ ), which is consistent with the present result. In fact, it would be difficult to distinguish between the result of $N$-body calculations and their stochastic FP result, except that their result is smoother while the central density is low.

Figure 2 shows the evolution of the number of particles in the core. For all runs, the number of particles at maximum contraction is of the order of 10 , while that at maximum expansion is $1-2 \%$ of the total number of particles. This result is again in good agreement with gas models and FP calculations.

Figure 3 shows the fraction of time for which the number of particles in the core is smaller than the value $N_{c}$ as a function of $N_{c} / N$, for the post-collapse phase. For $N>8192$, the median core mass is around 0.5$0.6 \%$. This corresponds to $r_{c} / r_{h} \sim 0.01$. For $N=32,768$, the core mass is 


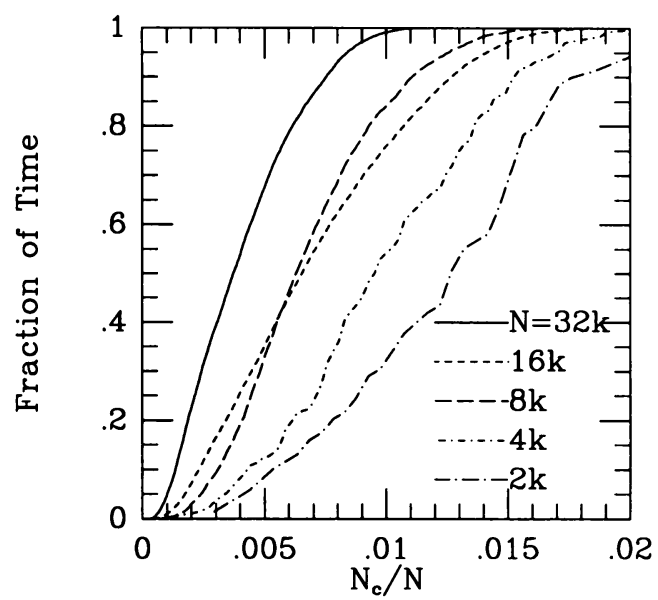

Figure 3. Fraction of time for which the number of particles in the core is smaller than $N_{c}$, as a function of $N_{c} / N$.

somewhat smaller than that for $16 \mathrm{k}$ or $8 \mathrm{k}$ runs. This difference is because the $32 \mathrm{k}$ run is not long enough.

Figure 3 indicates that the typical core mass for a post-collapse cluster would be less than $1 \%$, if gravothermal oscillation occurs. One interesting question is whether it is possible to distinguish a core in gravothermal oscillation from a core dominated by primordial binaries. The theoretical prediction by Goodman and Hut (1989) gives $r_{c} / r_{h} \sim 0.02$, while $N$-body simulations by McMillan et al. (1990) give $r_{c} / r_{h} \sim 0.2$. McMillan et al. (1990) used 1136 particles. If their $N$-body results can be extrapolated to a larger $N$, cores with primordial binaries and cores in gravothermal oscillation would be clearly distinguishable. To obtain a definitive answer, we need to perform simulations of clusters with primordial binaries using a larger number of particles than employed by McMillan et al. (1990).

\subsection{DETAILED VIEW OF THE 32K RUN}

In the previous section, we gave an overview of the post-collapse evolution of $N$-body point-mass systems with $2 \mathrm{k}-32 \mathrm{k}$ particles. In this section, we take a closer look of the 32k-particle simulation to see whether we can find a direct signature of the gravothermal expansion. It is generally believed that a long expansion phase without significant energy input and a temperature inversion during the long expansion phase are the most direct signatures of gravothermal expansion (Bettwieser and Sugimoto 1984, McMillan and Engle 1995). In this section we investigate both of them.

Figure 4 shows an enlarged view of the time variation of the core radius 

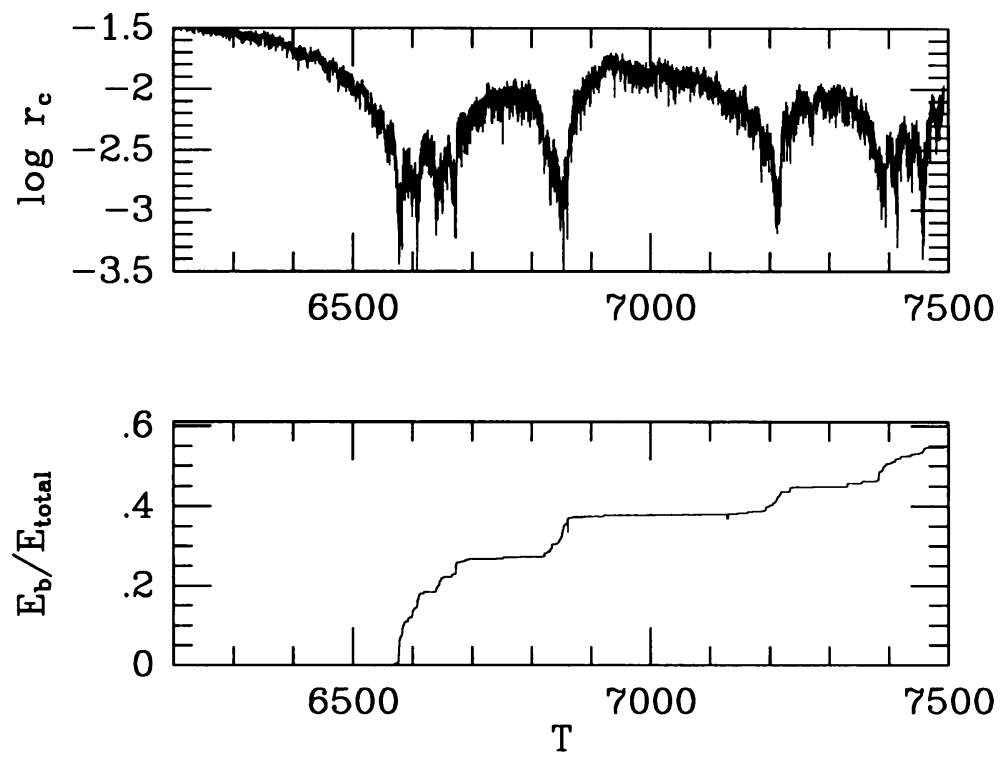

Figure 4. The core radius (top) and the binding energy of binaries (bottom) as a function of time for the $32 \mathrm{k}$-particle run

as compared with the sum of the binding energies of all binaries. It is clear that most of the energy is generated when the core is very small. Five peaks account for most of the energy production.

Continued expansion without energy generation is considered to be one of the most direct signatures of gravothermal oscillation. In figure 4 , we clearly see two such expansions, for $t=6700-6740$ and $t=6860-6920$. Both expansions continue for more than 10 half-mass crossing times, which is hundreds of the core relaxation time. These expansions cannot be driven simply by binary heating. If the expansion were driven only by binary heating, it could not continue without energy input for a timescale longer than the core relaxation time.

Figure 5 shows temperature profiles for the contracting and expanding phases. Near the end of the expanding phase a temperature inversion of the order of $5 \%$ is clearly visible. Since these profiles are time-averaged over 10 time units ( 80 snapshots), the actual inversion might be somewhat stronger. Note that the temperature inversion is visible only near the end of long expansion phases also in gas model and FP calculations (Bettwieser and Sugimoto 1984, Cohn et al. 1989).

Figure 6 shows the relation between the central density and the central velocity dispersion. The trajectory shows a striking resemblance to what is obtained by gas-model calculation (Goodman, 1987). The fact that the 

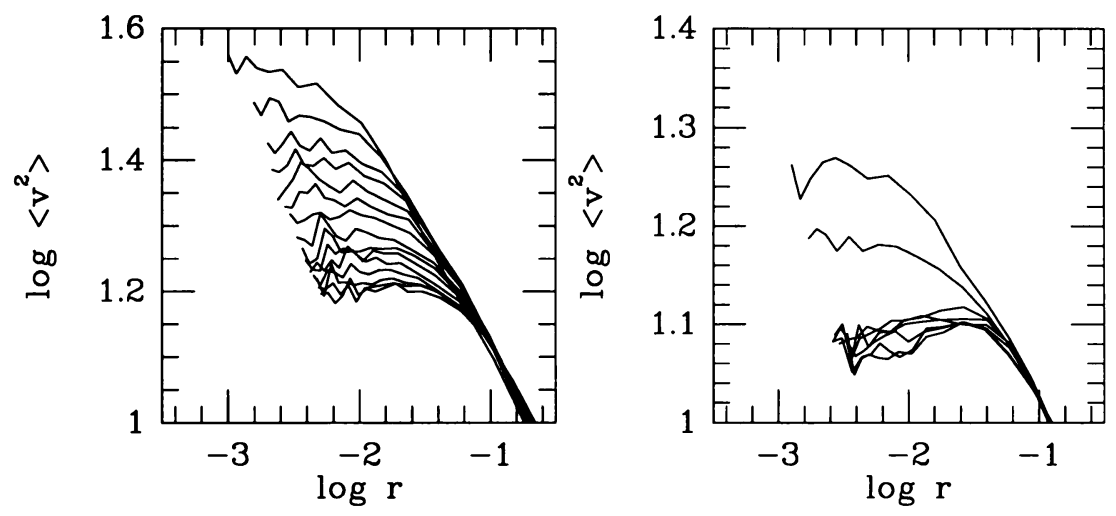

Figure 5. Velocity dispersion profiles for (a) contracting and (b) expanding phases. Each profile is obtained by time averaging over 80 snapshots (10 time units). The time interval between curves is 5 time units.

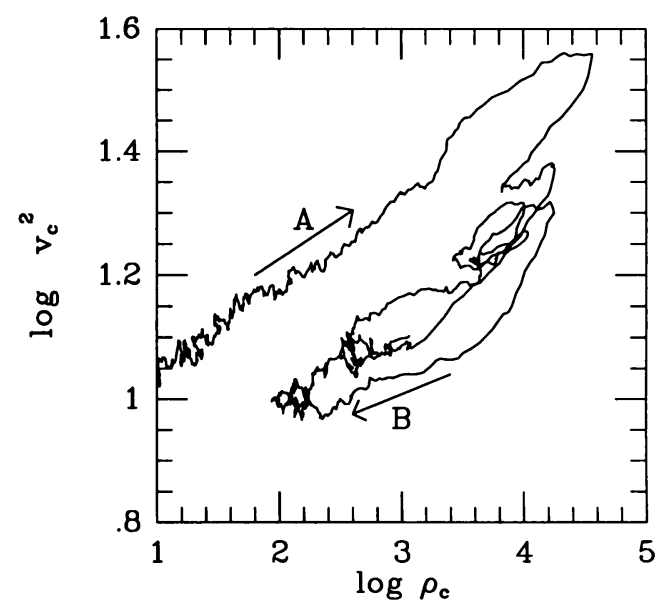

Figure 6 . The change of the central density and the central velocity dispersion. Each data points is time-averaged value over 80 snapshots. Arrows indicate the direction of evolution.

trajectory shows clockwise rotations means that this is a refrigeration cycle, in which the central region absorbs the heat when the temperature is low, and release heat when the temperature is high (Bettwieser and Sugimoto 1984, Bettwieser 1985). In particular, the later phase of the large expansions (indicated by the arrow marked "B") is nearly isothermal. Therefore this phase is driven by the heat supply. Since the binding energy of binaries is unchanged during this phase, the heat is supplied from outside the core. In other words, the expansion is gravothermal. 


\section{Discussion}

We have performed direct $N$-body simulation of the post-collapse evolution of globular clusters. We confirm that gravothermal oscillation actually takes place in a point-mass $N$-body system.

Whether real globular clusters undergo gravothermal oscillation or not is a question which requires further research. If clusters contain many primordial binaries, even after the core collapse they might still be burning the primordial binaries. In addition, the effect of two-body binaries on the evolution of the cluster is still unclear. In fact, the cross section for binary formation by tidal capture and for merging is not fully understood yet (Mardling 1995a, 1995b).

The most straightforward way to study the effect of primordial binaries or two-body capture is direct $N$-body simulation. In principle, we can put primordial binaries and their evolution into FP calculation by following the distribution of internal binding energy of binaries. However, the standard one-dimensional FP calculation, which assumes an isotropic distribution, is not appropriate for following the binary population, since most binaries are formed in the core and their orbits are nearly radial. Thus, we have to solve the FP equation at least in three dimensions $(E, J$ and the binary binding energy $E_{b}$ ). This would require prohibitively large computer power. One could also use the Monte-Carlo approach, but its result must be compared with $N$-body simulation anyway.

We have demonstrated that $N$-body simulation is now possible with a number of particles close to that in real globular clusters, thanks to the extremely powerful special-purpose computer and its full-time availability. We are now able to use direct $N$-body simulation to study various aspects of the evolution of globular clusters.

The present $32 \mathrm{k}$-particle calculation took about three months of CPU time on 1/4 of GRAPE-4. If we tried to do a similar calculation on a Cray T90 vector supercomputer, it would have taken several years of CPU time. This is simply impossible to do on present-day supercomputers. If we want to finish the calculation in, say, one CPU month, we need a computer 50100 times faster than a Cray T90, which will be available 10 years from now.

The CPU time of three months is still very long. However, for many simulations, we do not need as many as $32 \mathrm{k}$ particles. A $16 \mathrm{k}$-particle calculation was finished in 2-3 weeks, on $1 / 8$ of GRAPE-4. Thus to run many simulations of $16 \mathrm{k}$-particle systems is now practical.

If we can continue the development of the special-purpose computer, we will have a system 100-1000 times faster than the present GRAPE-4 in the next five years. Such a system will make it possible to run $50-100 \mathrm{k}-$ 
particle simulation routinely, while $500 \mathrm{k}-1 \mathrm{M}$-particle simulations will still take months.

\section{Acknowledgements}

It's a pleasure to thank Daiichiro Sugimoto for his effort on GRAPE project, and also for many useful discussions and comments on the manuscript. I'm grateful to Sverre Aarseth for his NBODY4 code, Makoto Taiji, Toshiyuki Fukushige and Toshikazu Ebisuzaki for developping GRAPE-4 along with myself, Piet Hut and Steve McMillan for many useful discussion and Ivan King for comments on the manuscript. This work was supported by the Grant-in-aid for Specially Promoted Research (04102002) of the Ministry of Education, Science, and Culture.

\section{References}

Aarseth, S. J. (1985) in Multiple Time Scales, eds. J. U. Brackhill and B. I. Cohen, Academic, New York, p. 377.

Bettwieser, E. (1985) in Dynamics of Star Clusters, IAU Symposium No. 113, eds. J. Goodman and P. Hut, Reidel, Dordrecht, p. 219.

Bettwieser, E. and Sugimoto, D. (1984) MNRAS 208, 493.

Casertano, S. and Hut, P. (1985) Ap. J. 298, 80.

Cohn, H., Hut, P., and Wise, M. (1989) Ap. J. 342, 814.

Giersz, M. and Heggie, D. (1994) MNRAS 268, 257.

Goodman, J. (1984) Ap. J. 280, 298.

Goodman, J. (1987) Ap. J. 313, 576.

Goodman, J. and Hut, P. (1989) Nature 339, 40.

Hachisu, I., Nakada, Y., Nomoto, K., and Sugimoto, D. (1978) Prog. Theor. Phys. 60, 393.

Heggie, D. (1986) in The Use of Supercomputers in Stellar Dynamics, eds. S. McMillan and P. Hut, Springer, New York, p. 233.

Heggie, D. and Ramamani, N. (1989) MNRAS 237, 757.

Heggie, D. , Inagaki, S., and McMillan, S.L.W. (1994) MNRAS 271, 706.

Kustaanheimo, P., and Stiefel, E. (1965) J. Reine. Angew. Math. 218, 204.

Lynden-Bell, D. and Eggleton, P.P. (1980) MNRAS 191, 483.

Makino, J. (1986) in The Use of Supercomputers in Stellar Dynamics, eds. S. McMillan and P. Hut, Springer, New York, p. 151.

Makino, J.. (1991) Publ. Astron. Soc. Japan 43, 841.

Makino, J. and Aarseth, S. J. (1992) Publ. Astron. Soc. Japan 44, 141.

Makino, J. and Sugimoto, D. (1987) Publ. Astron. Soc. Japan 39, 589.

Mardling, R. A. (1995a) Ap. J. 450, 722.

Mardling, R. A. (1995b) Ap. J. 450, 732.

McMillan, S. L. W. (1986) in The Use of Supercomputers in Stellar Dynamics, eds. S. McMillan and P. Hut, Springer, New York, p. 156.

McMillan, S. L. W. and Engle, E. A. (1995) in this volume.

McMillan, S. L. W., Hut, P. and Makino, J. (1990) Ap. J. 362, 522.

Spurzem, R. and Aarseth, S. J. (1996) preprint.

Sugimoto, D. and Bettwieser, E. (1983) MNRAS 204, 19p.

Takahashi, K. and Inagaki, S. (1991) Publ. Astron. Soc. Japan 43, 589.

Taiji, M. (1995) in this volume. 